\title{
Self-assembled biomimetic nanoreactors II: Noble metal active centres
}

\author{
Matt McTaggart $^{\mathrm{a}, \dagger}$, Cecile Malardier-Jugroot $^{\mathrm{a}}$, and Manish Jugroot ${ }^{\mathrm{b}}$ \\ ${ }^{a}$ Department of Chemistry and Chemical Engineering, ${ }^{b}$ Department of Mechanical and Aerospace Engineering, \\ Royal Military College of Canada, Kingston, ON, Canada, $K_{7} K_{7} B_{4}$
}

\begin{abstract}
The structure and stability of polymer-metal nanoreactors is detailed, including a complete characterization of the first successful synthesis of stable, dispersible, atomically thin gold nanosheets. Further developments in the synthesis of monodisperse, $\sim 2.5 \mathrm{~nm}$ platinum nanoclusters in aqueous solution and ambient conditions are described and $\mathrm{pH}$ stability of the composite material is established. The facile nanoreactor synthesis, environmentally friendly reaction conditions, and structural stability makes these biomimetic systems attractive for applications ranging from chemical detoxification to nanoelectronics.
\end{abstract}

Chemical processes of living organisms occur within nanostructured macromolecular reactors, enzymes, which employ both confinement and catalysis to control rates of reaction. The confinement effect describes the spontaneous rise in effective molarity of non-polar molecules within a hydrophobic nanoscale cavity ${ }^{1}$. Increased substrate concentration at the active site increases electron density and reduces the entropic cost, resulting in an increased reaction rate. The active centre comprises those components whose interactions with the substrate align to minimize activation energy; amino acid side groups typically stabilize intermediate reaction structures while metal centres co-ordinate electron transfer ${ }^{2}$. A similar combination of confinement and catalytic materials in an artificial nanoreactor is expected to open new applications for biomimetic systems.

The development of nanoscale metal catalysts whose size, morphology and properties of the particles could be controlled via designed synthesis is expected to have applications in a variety of high technology fields such as sensing, bioanalysis, biological labeling and semi-conductors. Several methods have been developed recently toward this goal including incipient wetness impregnation ${ }^{3}$, electron beam lithography $^{4}$, sol-gel ${ }^{5}$, co-precipitation ${ }^{6}$, and colloidal synthesis with block copolymers or dendrimers ${ }^{7,8}$. However these methods involve either precision fabrication or environmentally hazardous reaction conditions, and can reduce nanoparticle catalytic activity due to the surface passivity by stabilizing ligands ${ }^{9}$.

It was recently demonstrated that the hydrophobic salt platinum(II) chloride will form nanoclusters under $3 \mathrm{~nm}$ in dimension inside a poly(styrene-alt- maleic acid), SMA, nanostructure without additional reducing agents ${ }^{10}$. The polymer-supported metal nanoparticles are well dispersed and stable in the aqueous solvent and avoids precipitation of the complex often due to reducing agents. It was also shown that the platinum-SMA nanostructure could promote the polymerization of pyrrole through confinement and catalytic effects, mimicking biological systems ${ }^{10,11}$.

Gold is generally inert as a bulk material but nanocrystals under $5 \mathrm{~nm}$ have been found to demonstrate high catalytic activity ${ }^{12}$. Furthermore, gold nanosheets have been shown to form between layers of selfassembling surfactants ${ }^{13}$. The successful synthesis of platinum nanocrystals in this size domain within a self-assembling polymer encouraged expansion of the method to a hydrophobic gold salt. Gold with at least one dimension under $6 \mathrm{~nm}$ has a stable hexagonal close-packing ( $h c p)$ crystal structure that becomes dominant as that length decreases ${ }^{14}$, a novel crystal phase of interest for catalytic and optical applications.

Herein we report the theoretical and experimental characterization of the structure, and $\mathrm{pH}$ stability of SMA-platinum and SMA-gold nanoreactors. First, metal nanocrystal growth was measured by UV/Vis spectroscopy, which revealed a difference in reaction rate. Interactions of the metal atoms with the SMA polymer were then modeled by molecular simulation methods to expose the crucial role of the polymermetal interaction in the growth mechanism. The gold and platinum systems were further investigated by transmission electron microscopy (TEM) and in solution by small-angle neutron scattering (SANS). The nanoreactors obtained were produced by a facile and environmentally friendly reaction path and constitute very stable systems combining, like their biological counterpart, the effect of confinement with catalytic materials.

\section{Experimental}

Poly(styrene-alt-maleic acid) sodium salt solution, $13 \%$ wt in $\mathrm{H}_{2} \mathrm{O}, 350 \mathrm{kDa}$ average molecular weight (SMA, Sigma-Aldrich) was freeze dried and rehydrated according to each sample specification. Gold(I) chloride (AuCl, 99.9\%, Sigma-Aldrich) and platinum(II) chloride (PtCl2, 98\%, Sigma-Aldrich) were used as received. The synthesis method for all sam- 
ples follows that described previously ${ }^{10,15}$. In short, the requisite amounts of dried SMA polymer and hydrophobic metal salts were added to $3 \mathrm{~mL}$ of DI water and $\mathrm{HCl}$ or $\mathrm{NaOH}$ added to achieve the desired $\mathrm{pH}$ or, in the case of small-angle neutron scattering samples, $\mathrm{D}_{2} \mathrm{O}, \mathrm{DCl}$, and $\mathrm{NaOD}$. Mixing was completed by sonication for 0.5-1.5 hours. UV-visible light spectroscopy was carried out in a $1 \mathrm{~mm}$ quartz cuvette in an Agilent 8453 PDA spectrometer and analyzed with ChemStation software v.B.04.02. SANS experiments were performed on the NG3 $30 \mathrm{~m}$ instrument at the NIST Center for Neutron Research, Gaithersburg, $\mathrm{MD}$, as detailed in Part $\mathrm{I}^{15}$. The selected models for each spectrum obtained are detailed in the results and discussion section. The morphology of the metal particles was also studied by TEM on a high resolution FEI HR-TEM/STEM Titan 80-300LB microscope operating at $300 \mathrm{kV}$. Molecular modeling was completed using Gaussian 09 software ${ }^{16}$. Interactions between platinum atoms and the SMA trimer employed a 3-part ONIOM mode $1^{17}$. The outer monomers were included at the PM6 level to simulate the immediate chemical environment without high computational $\operatorname{cost}^{11}$. The central monomer and metal atoms were modeled by density functional theory using the B3PW91 hybrid functional ${ }^{19-21}$ with the 6-31G basis set for the main group elements and LanL2DZ for platinum and gold, the latter with double- $\xi$ type effective core pseudopotentials ${ }^{22}$. This arrangement has been shown to provide high quality results for simulating transition metal interactions with oxygen and benzene complexes ${ }^{23-25}$.

\section{Results and Discussion}

Platinum: As discussed in the introduction, to develop efficient and tailored nanoreactors that mimic biosystems, it is important to control the nature and the shape of the active centers to optimize their surface area and catalytic efficiency ${ }^{26}$.

In the current study, nanocluster synthesis was monitored by UV/visible light spectroscopy over 19 days to follow the reduction process of $\mathrm{PtCl}_{2}$ inside the confined environment of the polymeric template. The characteristic plasmon peak for platinum at $215 \mathrm{~nm}$ was hidden in the SMA absorption range so platinum reduction into nanoclusters was observed qualitatively by a change in solution colour from yellow to brown to black, shown in the spectra series of Figure 1 as the dissipation of the shoulder at $430 \mathrm{~nm}^{27-29}$. Completion of the synthetic process was indicated by stabilization of the signal after 14 days.

Platinum nanocrystals were previously observed at $\mathrm{pH} 7^{10}$ embedded in the polymer matrix suggesting that the platinum salt used for their synthesis concen-

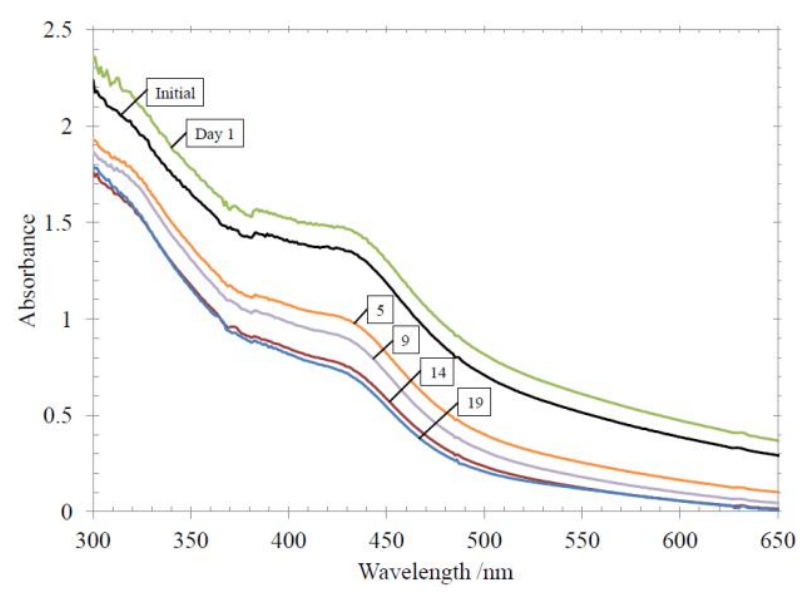

Figure 1. UV/vis spectra of $\mathrm{PtCl}_{2}$ in $1 \%$ wt SMA solution. Shoulder centred at $430 \mathrm{~nm}$ diminishes over time.

trates within SMA prior to its reduction. It is important, therefore, to understand the metal-polymer interactions since these determine whether the metal crystal growth is more likely to occur under confinement in the hydrophobic interior or on the hydrophilic exterior of the SMA nanostructure. When a platinum atom is placed at equal distance from the styrene and carboxylic acid moieties of the SMA monomer in DFT simulation, it follows the slope in the energy surface towards the styrene and forms bonds across two carbons of the ring; Figure 2 a shows its final geometry (hydrogen removed for clarity).

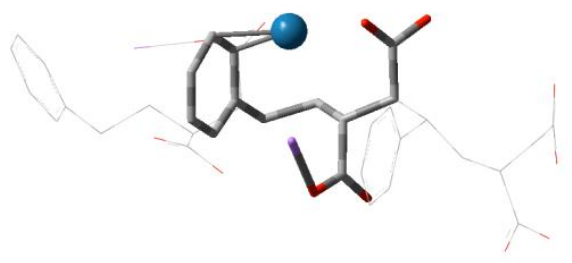

a) Pt atom bonded to styrene ring

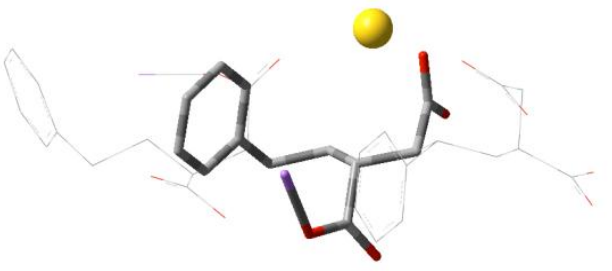

b) Au atom interacting with maleic acid

Figure 2. ONIOM (DFT-B3PW91/LanL2DZ/6-31G, PM6) optimized molecular simulation of a) platinum and b) gold atom coordination to a SMA trimer.

The metal-polymer bonding energy is calculated by the difference between the total system energy and that of the separate components:

$$
\mathrm{E}_{\text {bond }}=\mathrm{E}_{\text {SMA.Metal }}-\left[\mathrm{E}_{\mathrm{SMA}}+\mathrm{E}_{\text {metal }}\right]
$$

Bonding energy of the SMA-platinum bond shown in Figure $2 \mathrm{a}$ is $2.64 \mathrm{eV}\left(254.7 \mathrm{~kJ} \cdot \mathrm{mol}^{-1}\right)$. The stability of the styrene-platinum bond suggests that this is the nucleation site for nanocrystal formation. The loca- 
tion of the platinum nanoclusters within SMA's hydrophobic interior is in keeping with their $3 \mathrm{~nm}$ growth limit and catalytic effect on pyrrole polymerization. Simulations also show that the inclusion of the metal atom does not alter the linearity of the SMA polymer chain and should therefore leave the selfassembled nanoarchitecture formed by SMA unchanged $^{15,30}$.

Given the extensively studied $\mathrm{pH}$ sensitivity of the SMA self-assembly ${ }^{30,31}, \mathrm{pH}$ conditions might have affected platinum nanocluster growth within the template. Here, samples of the SMA-Pt nanoreactor system were prepared at $\mathrm{pH} 3.8,5,7$, and 9 and analyzed by HR-TEM. Micrographs displayed the presence of monodisperse platinum nanoclusters in each sample, positively identified by the crystal d-spacing and consistency with the results reported at $\mathrm{pH} 7^{10}$.
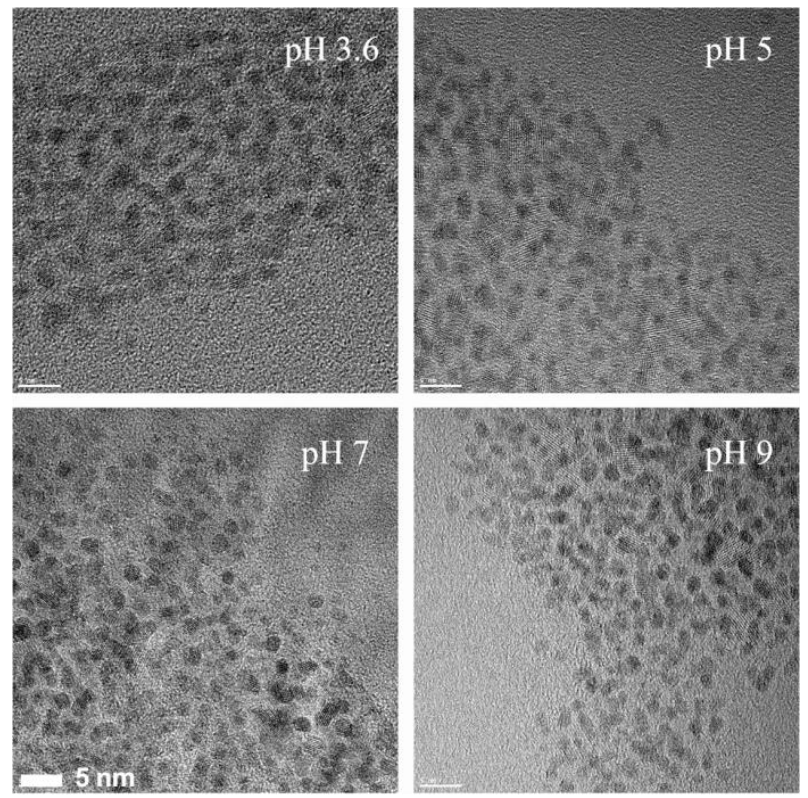

Figure 3. HR-TEM images of SMA-Pt systems prepared at $\mathrm{pH}$ levels shown. Scale bar is $5 \mathrm{~nm}$ in all images.

It is observed therefore that the SMA templated synthetic method is not $\mathrm{pH}$ dependent. Indeed, as seen in Figure 3, $\mathrm{pH}$ has no significant influence on the mean radius of samples prepared at $\mathrm{pH} 3.8(1.26$, s.d. $=0.10 \mathrm{~nm}), \mathrm{pH} 5(1.25$, s.d. $=0.12 \mathrm{~nm}), \mathrm{pH} 7(1.28$, s.d. $=0.10 \mathrm{~nm})$, or pH $9(1.27$, s.d. $=0.10 \mathrm{~nm})$.

Disruption of the SMA superstructure into individual polymer chains at low or high $\mathrm{pH}$ would cause precipitation of the platinum nanoclusters. The intact polymer superstructure with embedded Pt crystals is visible in HR-TEM images over the $\mathrm{pH}$ range investigated, indicating that the platinum-styrene interaction energy demonstrated by simulation allows the composite material to overcome the forces responsible for the $\mathrm{pH}$ sensitivity of SMA alone.

To characterize the SMA nanoreactors in solution, samples were analyzed by small-angle neutron scat- tering. The lamellar model for the SMA structure ${ }^{15}$ was combined with a triaxial ellipsoid form factor to model the metal nanocrystals:

$$
\begin{gathered}
P(q)=\frac{\text { scale }}{V_{\text {ell }}} \int_{0}^{1} \int_{0}^{1} \phi^{2}\left\{q \left[a^{2} \cos ^{2}\left(\frac{\pi x}{2}\right)\right.\right. \\
+b \sin ^{2}\left(\frac{\pi x}{2}\right)\left(1-y^{2}\right) \\
\left.\left.+c^{2} y^{2}\right]\right\} d x d y
\end{gathered}
$$

where

$$
\phi^{2}(x)=9\left(\frac{\sin x-x \cos x}{x^{3}}\right)^{2}
$$

This model was applied to the SANS data for SMAPt systems at $\mathrm{pH} 5$ and 7 with satisfactory results, as shown in Figure 4, demonstrating the $\mathrm{pH}$ stability of the composite in solution. The triaxial ellipsoid model parameters obtained from the fit of the spectra of the SMA-Pt system at $\mathrm{pH} 7$ and $\mathrm{pH} 5$ give and average diameter for the Pt nanoclusters of $2.5 \mathrm{~nm}$ for both $\mathrm{pH}$ values, shown in Table 1. This value is in agreement with the size observed by HR-TEM (Figure 3). The discrepancy between the data and fit in the low $\mathrm{Q}$ range of the SMA-Pt systems is attributed to the laminar form factor's assumption of an infinite sheet where SMA structures are finite within this range.

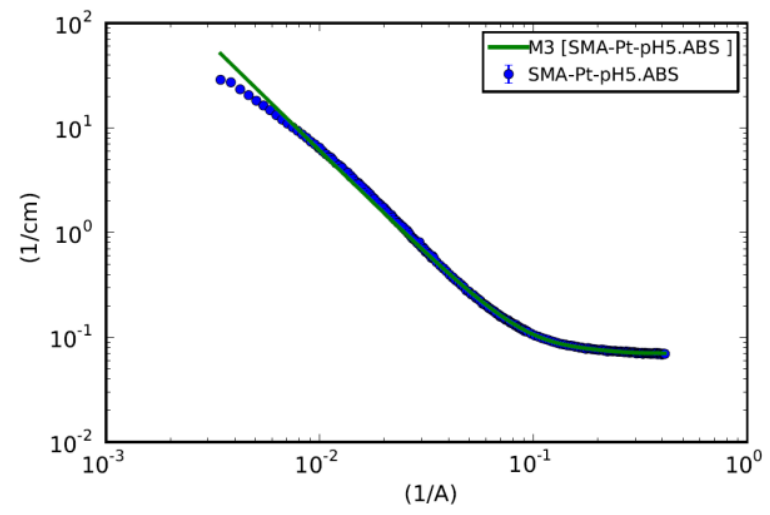

a) $\mathrm{pH} 5$

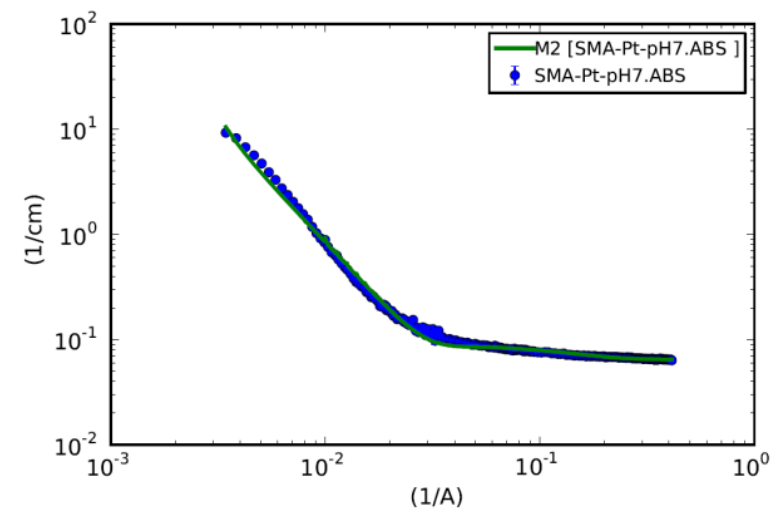

b) $\mathrm{pH} 7$

Figure 4. Lamella-ellipsis fit (line) to SANS data of $\mathrm{PtCl}_{2}$ in $1 \%$ SMA at a) pH 5 and b) pH 7. 
Table 1. SANS model fitting parameters.

\begin{tabular}{|c|c|c|c|c|c|c|}
\hline & Parameter & SMA pH 7 & SMA-Au pH7 & SMA-Pt pH7 & SMA-Pt pH5 & \\
\hline \multirow{5}{*}{. } & $\mathrm{SLD}_{\text {solid }}$ & $1.87 \mathrm{e}-06$ & $1.87 \mathrm{e}-06$ & $1.87 \mathrm{e}-06$ & $1.87 \mathrm{e}-06$ & \\
\hline & $\mathrm{SLD}_{\text {solv }}$ & $6.38 \mathrm{e}-06$ & $6.38 \mathrm{e}-06$ & $6.38 \mathrm{e}-06$ & $6.38 \mathrm{e}-06$ & \\
\hline & Spacing $s(\AA)$ & 6.99 & 8.04 & 6.66 & 6.30 & \\
\hline & Thickness $t(\AA)$ & 12.34 & 95.64 & 14.59 & 12.61 & \\
\hline & Layers $n(\mathrm{~N})$ & 1.00 & 7.23 & 1.01 & 5.38 & \\
\hline \multirow{5}{*}{ 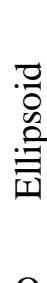 } & $\mathrm{SLD}_{\text {solid }}$ & & $4.66 \mathrm{e}-06$ & $6.34 \mathrm{e}-06$ & $6.34 \mathrm{e}-06$ & \multirow{6}{*}{$\begin{array}{l}\text { Schematic representations } \\
\text { of the form factors used in } \\
\text { SANS fitting for lamina and } \\
\text { tri-axial ellipsoid. }\end{array}$} \\
\hline & $\mathrm{SLD}_{\text {solv }}$ & & $6.38 \mathrm{e}-06$ & $1.87 \mathrm{e}-06$ & $1.87 \mathrm{e}-06$ & \\
\hline & Radius $a$ & & 2.68 & 16.76 & 7.97 & \\
\hline & Radius $b$ & & 162.04 & 7.87 & 14.61 & \\
\hline & Radius $c$ & & 266.78 & 13.69 & 14.74 & \\
\hline \multicolumn{2}{|c|}{ Quality of fit $\left(x^{2} / n\right)$} & 37.071 & 3.749 & 18.483 & 85.763 & \\
\hline
\end{tabular}

It is interesting to note that the scattering length density of platinum and $\mathrm{D}_{2} \mathrm{O}$ are nearly identical, meaning that free platinum particles would have such low contrast to solution as to be invisible to SANS. In the fitted model, however, using SMA rather than $\mathrm{D}_{2} \mathrm{O}$ as the solvent for the nanoclusters gives a better fit. This is in agreement with the simulation results showing platinum's preference for the hydrophobic region as well as the TEM series showing the nanoclusters embedded within the SMA structure, giving further evidence that platinum nanocluster synthesis occurs within the confined hydrophobic interior of the SMA structure and remain there at lower $\mathrm{pH}$.

\section{Gold:}

Gold(I) chloride reduction and crystallization in the polymer template without reducing agents was monitored by UV/Vis, shown in Figure 5 by the growth of its characteristic peak at $547 \mathrm{~nm}$. The peak is seen to increase for the first 9 days and then settle slightly.

The diameter of spherical gold colloids in water can be closely estimated by the correlation of its radius to the wavelength of its plasmon band in accordance with Mie's theory ${ }^{32}$. Initial analysis by this method suggested a gold nanocrystal diameter of $77.7 \mathrm{~nm}$, far greater than SMA's $2.8 \mathrm{~nm}$ limiting inner cavity. This first suggested different reaction mechanisms and final structures for gold and platinum in SMA solution. The Mie-based radius calculation method assumes free spherical particles-conditions that we will find are not valid in this case.

Molecular simulations (DFT) were performed to predict the fine polymer-gold interactions. Beginning in identical position relative to the SMA polymer as the platinum atom, a free gold atom was found to preferentially associate with oxygen-carrying functional groups. Energy minimization pulls gold away from the hydrophobic regions and toward the nearest oxygen for a binding energy of $0.21 \mathrm{eV}(19.95 \mathrm{~kJ}$ $\left.\mathrm{mol}^{-1}\right), 1 / 10$ th the Pt-styrene association strength (Figure 2b). This indicates that gold nanocrystal formation should occur primarily on the hydrophilic surface of the polymer structure and gives further indication that platinum and gold salt reduction proceeds along different pathways. As with platinum, the gold atom does not disrupt the polymer chain's linear conformation and so should not affect SMA selfassembly.

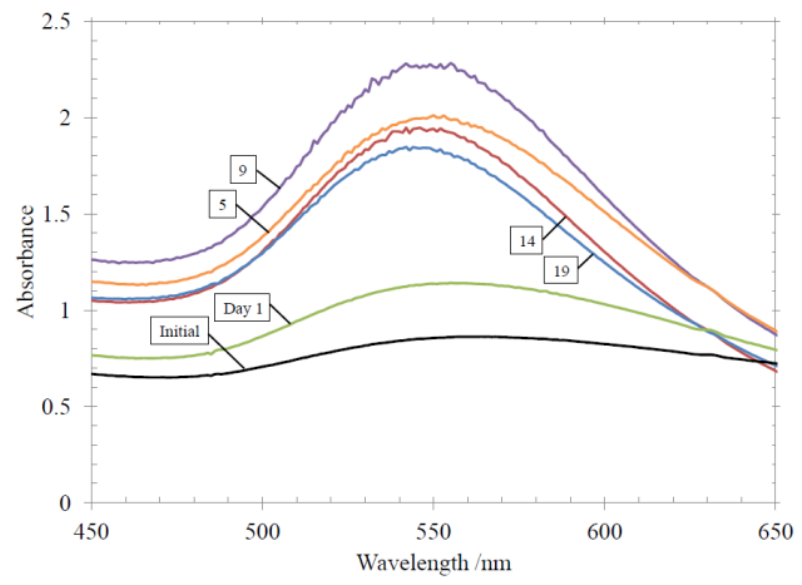

Figure 5. UV/vis spectra of $\mathrm{AuCl}$ in 1\% SMA solution. Peak centred at $547 \mathrm{~nm}$ increases for 9 days and becomes steady.

In our current study, HR-TEM images of goldSMA systems reveal that single crystal gold monolayers were produced (Figure 6a). A selected area electron diffraction (SAED) pattern taken from the crystal shown in Figure 6 indexes to the (0001) $h c p$ 
plane (inset). Calculating the radial distance of the $1 \overline{2} 10$ and $1 \overline{1} 00$ points using the unit cell parameter $a$ $=2.96 \AA$, in accordance with Huang ${ }^{14}$, places those points at $7.94 \mathrm{~nm}^{-1}$ and $4.20 \mathrm{~nm}^{-1}$ respectively - distances seen exactly on the SAED pattern. The $d$ spacing associated with these two points are $0.14 \mathrm{~nm}$ $(1 \overline{2} 10)$ and $0.24 \mathrm{~nm}(1 \overline{1} 00)$, both of which are measurable in TEM images.

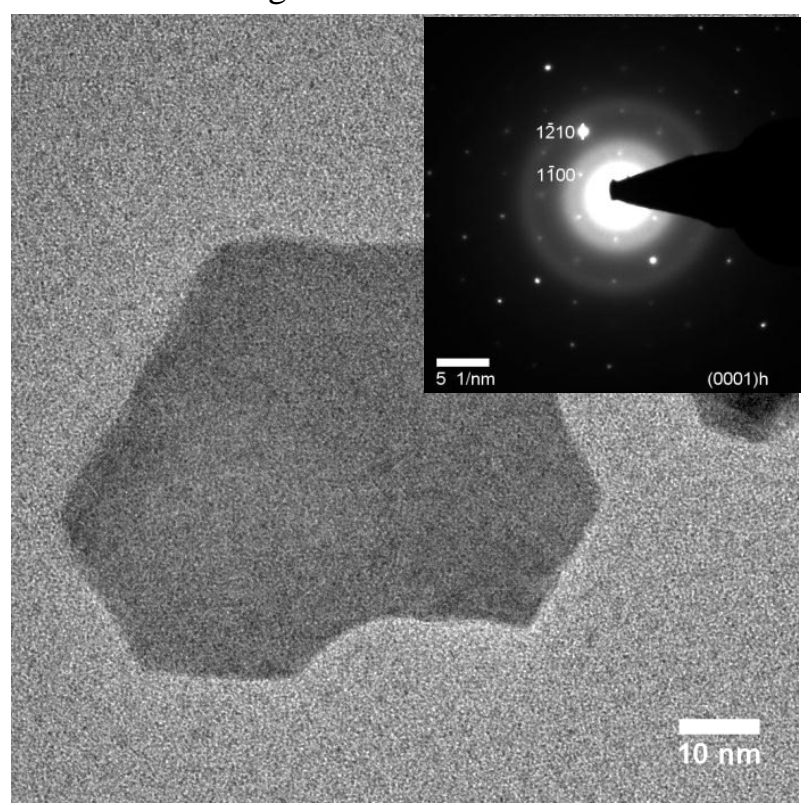

a) $2.6 \mu \mathrm{m}^{2}$ gold monolayer. Scale bar is $10 \mathrm{~nm}$. Inset: SAED is consistent with hexagonal close-packing crystal structure.

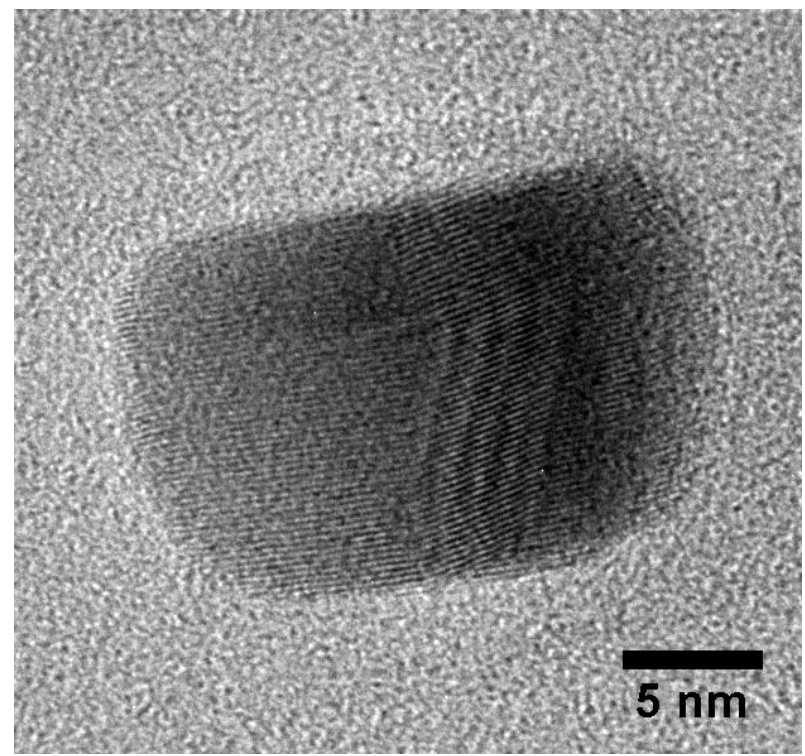

b) Two discrete gold nanosheets producing a $1 \mathrm{~nm}$ Moiré fringe pattern by their overlap. Scale bar is $5 \mathrm{~nm}$.

Figure 6: TEM images of atomically flat gold monolayers

Unlike the previous study of $h c p$ in gold nanosheets ${ }^{7}$, our results do not show evidence of phase transition to face-centred cubic $(f c c)$ under electron beam irradiation. Nanosheet thickness on the order of single atomic layers explains their stability since
(0001) $h c p$ and the (111) fcc plane are identical at that scale. Their stability as independent monolayers is also evident by the appearance of Moiré fringe patterns where there is overlap of two monolayers, as shown in Figure 6b. These interference patterns arise where one layer of parallel lines is visible through another. The distance $d$ between peaks of Moiré fringes is $d=s / \sin \alpha$ where $s$ is the underlying line spacing and $\alpha$ is the angle between them. In this case $s=0.24 \mathrm{~nm}$ and $\alpha=14^{\circ}$, making $d=1 \mathrm{~nm}$, equal to the band distance observed, giving strong evidence of the successful synthesis of monatomic gold layers.

There is a tendency, however, for the gold plates to cluster and aggregate, as seen in Figure $6 \mathrm{~b}$. This could be an artefact of the drying process though it could also be evidence that SMA-Au association produces alternating layers of polymer and metal. This conformation would place islands of atomically thin and flat $h c p$ gold within a hydrophilic confined space, an arrangement with exceptionally high potential for catalytic application.

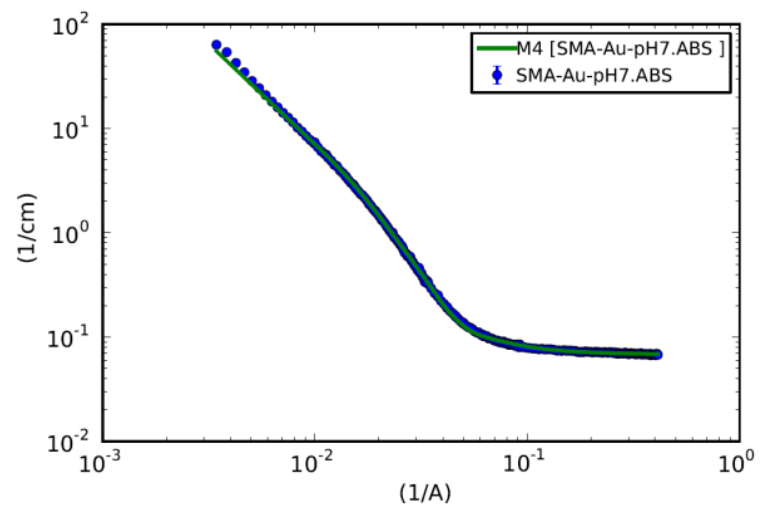

Figure 7. Lamella-ellipsis model fitted to SANS data of $\mathrm{AuCl}$ in $1 \%$ SMA, pH 7.

The SANS analysis performed provided further evidence of gold monolayer formation within the hydrophilic confinement space created by adjacent SMA bilayers. The same lamella-ellipsoid model as was used for SMA-Pt was fitted to the SMA-Au system. The three radii parameterized by $a, b$, and $c$ fit well where $a=2.7 \AA, b=16.2 \mathrm{~nm}$ and $c=26.6 \mathrm{~nm}$, consistent with an atomically thin gold disk (Figure 7). These data confirm correspondence between the crystalline polymer sheets and gold monolayers seen under TEM with their existence in solution. The thickness parameter optimized to $95.6 \AA$ in 7 layers, sup-

porting the hypothesis that the clustering of gold monolayers is due to SMA-Au layering in solution (Table 1).

To the best of our knowledge, this is the first successful synthesis of stable, dispersible, atomically thin gold nanosheets in ambient conditions. Recently reported syntheses using $\mathrm{HAuCl}_{4}$ without additional 
reducing agent have produced gold nanosheets under $10 \mathrm{~nm}$ but none thinner than $2.4 \mathrm{~nm}^{13,14,32-35}$. The results presented here extend those findings to include a simple and environmentally friendly synthesis of single atomic layer gold sheets by $\mathrm{AuCl}$ in SMA. This first successful synthesis of pure atomically thin gold sheets holds great promise for advances in electronics, sensing, medicine and plasmonics ${ }^{36,37}$.

To explore the interdependence of confinement domain and active centre metal, experimental sets were prepared to compare two methods of mixing gold and platinum salts together in SMA solution: one by mixing $\mathrm{PtCl}_{2}$ and $\mathrm{AuCl}$ together and adding the mixture to $3 \mathrm{~mL}$ of SMA solution (Method 1), the other by mixing $\mathrm{PtCl}_{2}$ in $1.5 \mathrm{~mL}$ SMA solution and $\mathrm{AuCl}$ in $1.5 \mathrm{~mL}$ solution and combining the two into a single container after sonication (Method 2).

Following the evolution of synthesis of platinum and gold nanoparticles by UV/vis spectroscopy provided insight into mechanistic and structural differences specific to the metal salt species and mixing method. The UV/vis spectra obtained for Method 1, Figure $8 \mathrm{a}$, is not equivalent to a combination of those for platinum and gold shown in Figure $8 \mathrm{a}$ and $8 \mathrm{~b}$ instead it appears to produce some electronic interaction between the metal crystals. The decreased intensity of the gold peak and red-shift to $554 \mathrm{~nm}$ in Figure $8 \mathrm{a}$ is indicative of gold-platinum alloying or the formation of a gold-platinum core shell structure ${ }^{38,39}$. A red shift greater than $20 \mathrm{~nm}$ combined with lower intensity has been reported for a 3:1 Au:Pt ratio ${ }^{38}$. The relative subtlety of the effect observed here means that there would be a very small amount of platinum in either arrangement.

However, because the intensity of the gold peak is pulled downward by platinum reduction between day 5 and 9 it is more likely that nanocrystal synthesis occurs independently for each metal and that they might interact indirectly through the polymer sheet.

The last sample is a combination of separately prepared platinum and gold SMA systems mixed by Method 2. The spectrum in Figure $8 \mathrm{~b}$ is a sum of the separate gold and platinum peaks indicating independent crystal growth. It also suggests no exchange of metals between the SMA-Pt and SMA-Au systems will occur. Metal-SMA association is rapid and the polymer-crystal bond is highly stable once formed.

In the samples prepared with gold and platinum salts in solution, there was no evidence seen of alloy formation, although the possibility that some platinum atoms are included in the gold crystals cannot be ruled out. Since the size and shape of the platinum nanoclusters are unchanged, the two metals appear to crystallize independently of one another: platinum

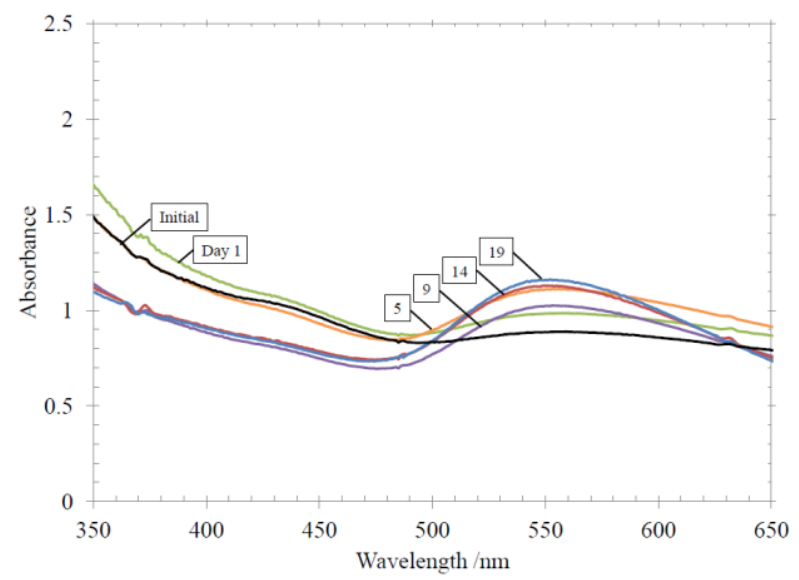

a) Shoulder at $430 \mathrm{~nm}$ diminishes and peak at $554 \mathrm{~nm}$ increases over time.

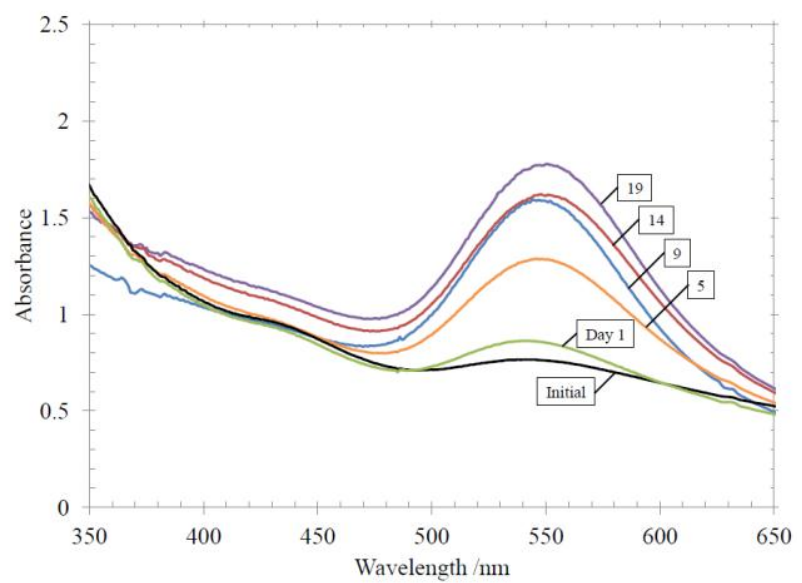

b) Peak is centred at $547 \mathrm{~nm}$ and rises with time.

Figure 8: $\mathrm{UV} /$ vis spectra of $\mathrm{PtCl}_{2}$ and $\mathrm{AuCl}$ samples mixed by a) method 1 and b) method 2 .

within and gold on the exterior surface of stacked SMA bi-layers.

This arrangement has a strong potential to produce a nanoreactor system capable of catalyzed and confinement-enhanced reactions of both polar and nonpolar substrates. Controlling the hydrophobicity is a crucial parameter in optimizing a nanoreactor as it controls the physical properties of the solvent (diffusion, density, structure, transport properties) ${ }^{40}$. Therefore, being able to control reactions within confined cavities with different hydrophobicity and different active centers within a nanoreactor holds great promise for controlled reactions under environmentally friendly conditions. The affinity of gold toward the hydrophilic cavity and Pt toward the hydrophobic cavity of SMA using hydrophobic precursors for the synthesis of the active centers $\left(\mathrm{AuCl}\right.$ and $\mathrm{PtCl}_{2}$ respectively) emphasizes the crucial role of the poly- 
mer-metal interaction for the optimization of the catalytic centre.

\section{Conclusion}

Platinum(II) chloride will preferentially concentrate within hydrophobic nanodomains of the SMA architecture to produce nanoclusters with a diameter $<3 \mathrm{~nm}$. This reaction occurs over a very broad $\mathrm{pH}$ range through a simple, reliable, and environmentally friendly synthetic method. When the same method is applied to gold(I) chloride, however, it generates pure, stable $h c p$ monolayers through the preferential interaction of gold with the hydrophilic surfaces of the SMA nanostructure. This first successful synthesis of pure atomically thin gold sheets holds great promise for advances in electronics, sensing, medicine and plasmonics.

Characterization of the structure and catalytic efficiency of systems containing active centres in stacked nanodomains of alternating hydrophilicity remains an active area of investigation. The self-assembling tertiary nanostructures suggested by the spectroscopic studies reported here may allow the development of a hardy and versatile nanoreactor.

The fact that the laminar SMA-metal nanoreactor structure remains stable in low $\mathrm{pH}$ conditions opens the possibility of application in a range of environmental conditions, such as the acidic electrolyte of proton exchange membrane fuel cells or in the variable $\mathrm{pH}$ and ion strength of water detoxification.

\section{AUTHOR INFORMATION}

\section{Corresponding Author}

Prof. Cecile Malardier-Jugroot

cecile.malardier-jugroot@rmc.ca

\section{Funding Source}

Natural Sciences and Engineering Research Council of Canada (NSERC) Discovery Grant.

\section{ACKNOWLEDGMENT}

The authors would like to thank NSERC for their generous support. We thank Dr. A. Korinek for his assistance at the Canadian Centre at the Electron Microscopy, McMaster University and Dr. B. Hammouda for SANS experiments performed at the National Center for Neutron Research, National Institute of Standards and Technology (NIST). This work is based upon NIST-CNR measurements supported in part by the National Science Foundation under Agreement DMR-0944772. This work benefitted from SasView software, originally developed by the DANSE project under NSF award DMR-0520547.

\section{REFERENCES}

(1) M. Raynal, P. Ballester, A. Vidal-Ferran, and P.W.N.M. van Leeuwen, Chem. Soc. Rev. 2014, 43, 1734-1787.

(2) X Zhang and K.N. Houk, Acc. Chem. Res. 2005, 38, 379-385.

(3) F. Auprêtre, C. Descorme, D. Duprez Catal. Commun., 2002, 3, 263

(4) . Grunes, J. Zhu, E.A. Anderson, G.A. Somorjai, J. Phys. Chem. B, 2002, 106,11463.

(5) A. Kaddouri, C. Mazzocchia, Catal. Commun., 2004, 5, 339.

(6) A.J. Akande, R.O. Idem, A.K. Dalai, Appl. Catal., A, 2005, 287, 159.

(7) G. Somorjai, J. Park, Top. Catal., 2008, 49, 126.

(8) R.M. Crooks, M. Zhao, L. Sun, V. Chechik, L.K. Yeung, Acc. Chem. Res., 2000, 34, 181.

(9) Y. Li and M.A. El-Sayed, J. Phys. Chem. B, 2001, 105, 8938-8943. (10) X. Li and C. Malardier-Jugroot, Macromol. 2013, 46, 2258-2266.

(11) M.N. Groves, C. Malardier-Jugroot and M. Jugroot, Chem. Phys. Lett. 2014, 612, 309-312.

(12) T. Fujita, P. Guan, K. McKenna, X. Lang, A. Hirata, L. Zhang, M. Chen, Nature materials 2012, 11(9), 775-780.

(13) H.L. Qin, D. Wang, Z.L. Huang, D.M. Wu, Z. C. Zeng, B. Ren, K. $\mathrm{Xu}$ and J. Jin, J. Am. Chem. Soc. 2013, 135, 12544-12547.

(14) X. Huang, S. Li, Y. Huang, S. Wu, X. Zhou, S. Li, C.L. Gan, F. Boey, C. A. Mirkin and H. Zhang, Nat. Comms. 2011, 2, 292.

(15) M. McTaggart, C. Malardier-Jugroot and M. Jugroot, Chem. Phys Lett. 2015, XXX, XXX-XXX. (Part 1)

(16) Gaussian 09, Revision B.01, M.J. Frisch, G.W. Trucks, H. Schlegel at al., Gaussian, Inc., Wallingford CT, 2009.

(17) M. Svensson, S. Humbel, R.D. Froese, T. Matsubara, S. Sieber and K. Morokuma, J. Phys. Chem., 1996, 100, 19357-19363.

(18) J.J. Stewart, J. Mol. Modeling, 2007, 13, 1173-1213.

(19) A.D. Becke, J. Chem. Phys. 1993, 98, 5648-5652.

(20) J.P. Perdew, J.A. Chevary, S.H. Vosko, K.A. Jackson, M.R. Pederson, D.J. Singh and C. Fiolhais, C. Phys. Rev. B: Condens. Matter 1992 46, 6671-6687.

(21) J.P. Perdew, and Y. Wang, Phys. Rev. B: Condens. Matter 1992, 45, 13244-13249.

(22) P.J. Hay and W.R. Wadt, J. Chem. Phys. 1985, 82, 270-283.

(23) T. Li and P. Balbuena, J. Phys. Chem. B 2001, 105, 9943-9952.

(24) E.S. Kryachko, A.V. Arbuznikov and M.F.A. Hendrickx, J. Phys. Chem. B 2001, 105, 3557-3566.

(25) T.C. Shore, D. Mith, D. DePrekel, S. McNall and Y. Ge, Reac Kinet. Mech. Cat. 2013, 109, 315-333.

(26) L. Ruan, H. Ramezani-Dakhel, C. Lee, Y. Li, X. Duan, H. Heinz and Y. Huang, ACS Nano, 2014, 8 (7), 6934-6944.

(27) Q.Y. Deng, B. Yang, J.F. Wang, C.G. Whiteley and X.N. Wang, Biotechnol. Lett. 2009, 31, 1505-1509.

(28) J. Huang, C. He, X. Liu, Y. Xiao, K.Y. Mya and J. Chai, Langmuir 2004, 20, 5145-5148.

(29) Z. Liu, X.Y. Ling, X. Su and J.Y. Lee, J. Phys. Chem. B 2004, 108, 8234-8240.

(30) C. Malardier-Jugroot, T.G.M. Van de Ven and M.A. Whitehead, J. Phys. Chem. B 2005, 109, 7022-7032.

(31) C. Malardier-Jugroot, T.G.M. van de Ven, T. Cosgrove, R. Richardson and M.A. Whitehead, Langmuir 2005, 21, 10179-10187. (32) W. Haiss, N.T.K. Thanh, J. Aveyard and D.G. Fernig, Anal. Chem. 2007, 79, 4215-4221.

(33) H. Ataee-Esfahani, L. Wang, Y. Nemoto and Y. Yamauchi, Chem. Mater. 2010, 22, 6310-6318.

(34) C. Vargas-Hernendez, M.M. Marsical, R. Esparza and M.J. Yacaman, Appl. Phys. Lett. 2010, 96, 213115.

35) Y. Shang, C. Min, J. Hu, T. Wang, H. Liu and Y. Hu, Solid State Sci. 2013, 15, 17-23.

(36) P.K. Jain, X. Huang, I.H. El-Sayed and M.A. El-Sayed, Acc. Chem. Res. 2008, 41, 1578 .

(37) A. Manjavacas and F.J. Garcia de Abajo, Nat. Comms. 2014, 5, 3548.

(38) N. Toshima and T. Yonezawa, New J. Chem. 1998, 22, 1179-1201.

(39) M. Bahram, F. Hoseinzadeh, K. Farhadi, M. Saadat, P. Najafi-

Moghaddam and A. Afkhami, Colloids and Surfaces A: Physicochem. Eng Aspects, 2014, 441, 517-524.

(40) J. L. Trick, E. J. Wallace, H. Bayley and M. S. P. Sansom, ACS Nano, 2014, 8 (11), 11268-11279 


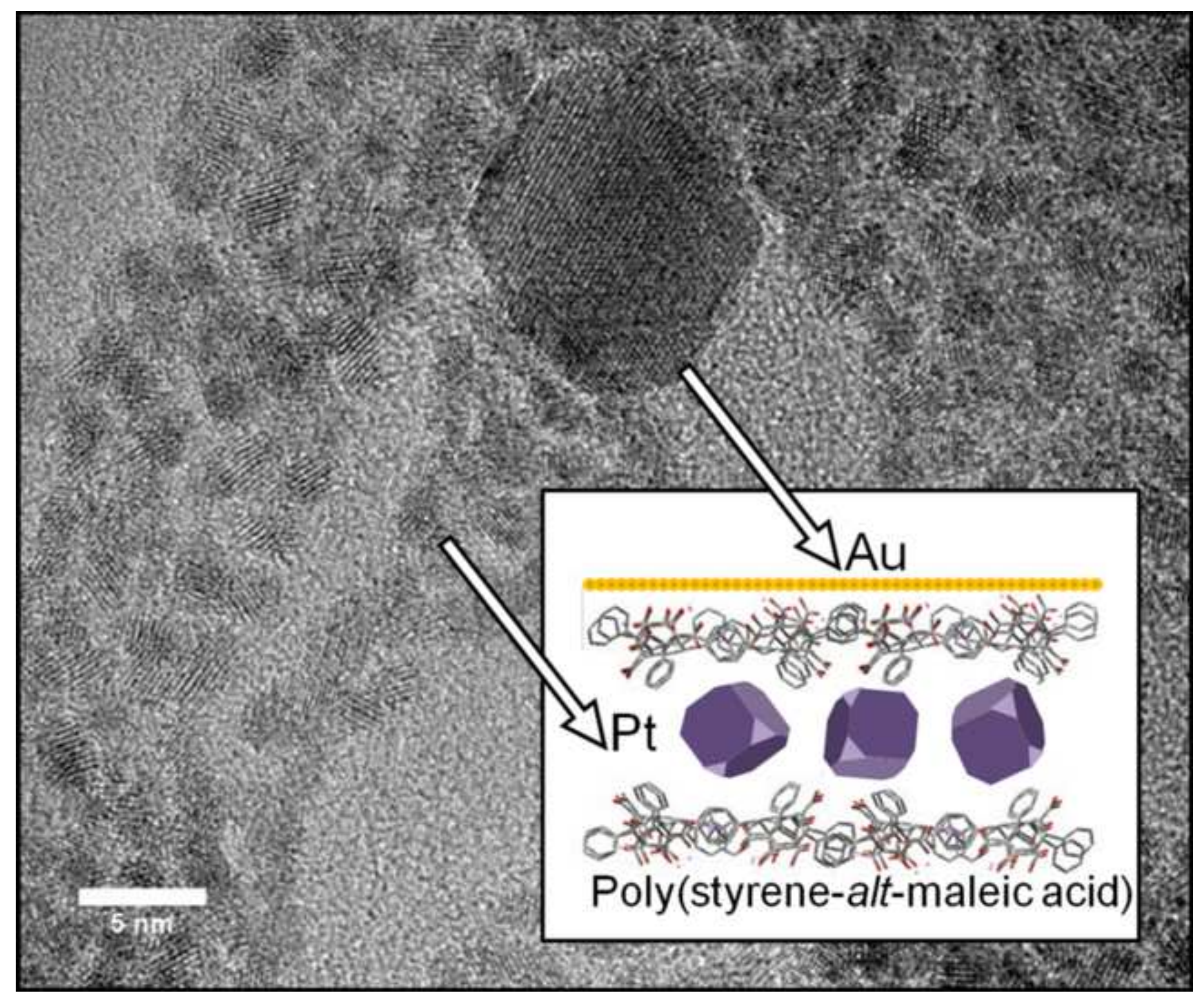

